\title{
Uso de la serie el Profesor Súper Ó para el desarrollo de competencias comunicativas ${ }^{1}$
}

\section{Use of the series the super teacher O for the development of communicative competitions}

DOI: http://dx.doi.org/10.17981/cultedusoc.9.3.2018.81

Artículo de investigación. Fecha de recepción: 15/06/2018. Fecha de aceptación: 27/11/2018

Iván Camargó2;

Teófila Pomarico; Alba Venera; Carmen Vega; Lercery Patricia y Tulia Salazar ${ }^{3}$

Institución Educativa Departamental Félix Ospino (Colombia)

ivancamargo20@gmail.com

Para citar este artículo:

Camargo, I., Pomarico, T., Venera, A., Vega, C., Patricia, L. y Salazar, T. (2018). Uso de la serie el Profesor Súper Ó para el desarrollo de competencias comunicativas. Cultura. Educación y Sociedad 9(3), 691-698. DOI: http://dx.doi.org/10.17981/cultedusoc.9.3.2018.81

\section{Resumen}

En la actualidad el sistema educativo ha experimentado transformaciones de manera significativa debido a la globalización y aspectos que se han considerado importantes implementar en los nuevos procesos de enseñanza como lo son el uso de estrategias pedagógicas innovadoras que apunten a dinamizar el plan curricular. Por tal razón, el estudio tiene como objetivo mejorar la expresión oral y escrita en el área de Lengua Castellana de los estudiantes de la Institución Educativa Félix Ospino, implementando las Tecnologías De La Información y Comunicación. La metodología se orientó desde un tipo de investigación cualitativa desarrollada desde la investigación como estrategia pedagógica (IEP), utilizando técnicas como la observación e instrumentos de recolección como el diario de campo. Se contó con una población participante de cincuenta (50) estudiantes. Los resultados evidenciaron que utilizar estrategias didácticas en el aula de clases permite que los estudiantes interactúen mayormente con los docentes y favorece el aprendizaje significativo, donde el uso de las TIC articuladas con la investigación, potencializan nuevas habilidades no adquiridas con las cátedras magistrales.

Palabras clave: tecnologías de la información y la comunicación, competencias comunicativas.

\section{Abstract}

At present the educational system it has experienced transformations of a significant way, due to the globalization and aspects that have been considered to be important to help in the new processes of education like it is the use of pedagogic innovative strategies that point to stir the plan into action curricular, for such a reason the study Félix Ospino has as aim improve the oral and written expression of the students of the Educational Institution in the area of Castilian Language implementing the technologies of the information and communication. The methodology was orientated from a type of qualitative investigation developed from the investigation as pedagogic strategy (IEP), using technologies as the observation and the field diary, possessing a population participant of fifty (50) students. The results demonstrated that to use didactic strategies in the classroom of classes, it is allowed that the students should interact with the teachers and to favor the significant learning, where on having implemented the use of the TIC, articulated to the investigation it promotes new skills not acquired with the magisterial chairs.

Keywords: information and communication technologies, communication skills.

1 Este artículo ha sido derivado del Programa de Fortalecimiento de la Cultura Ciudadana y Democrática CT+I a través de la IEP apoyada en TIC en el Departamento de Magdalena: PROYECTO CICLON

2 Líder del grupo de investigación "La Vecindad del Félix Ospino".

3 Docentes de la Institución Educativa Departamental Félix Ospino y miembros del grupo investigación "La Vecindad del Félix Ospino".

- The author; licensee Universidad de la Costa - CUC.

Cultura, Educación y Sociedad vol. 9 no. 3, pp. 691-698. Diciembre, 2018

Barranquilla. ISSN 2389-7724 Online 


\section{Introducción}

Partiendo del diagnóstico previo realizado por docentes de la Institución Educativa Félix Ospino Mugno, se apunta a intervenir en temas relacionados con el mejoramiento de la expresión escrita y oral a través de las TIC, debido a que se manifiestan falencias en procesos de lectura y escritura, principalmente en el área de Lengua Castellana. Sin embargo, se evidencia también la afectación en el rendimiento académico en otras áreas de formación escolar.

Así mismo, se identifica que los estudiantes interactúan mayormente con aparatos tecnológicos propios de la contemporaneidad (Herrera-Mendoza, Acuña, y Gil, 2017), lo que ha llevado a los docentes a considerar que sería de gran utilidad implementar estrategias pedagógicas basadas en las TIC para obtener una intervención efectiva en cuanto a la asimilación y mejora de habilidades y competencias en el área de Lengua Castellana.

$\mathrm{El}$ sistema educativo, a partir de la globalización, ha implementado nuevas maneras de comunicación y por ende de socializar los conocimientos que se adquieren en el proceso de enseñanza y de aprendizaje. Partiendo de lo anterior, el maestro y el estudiante han innovado su relación cotidiana implementando nuevos elementos como las Tecnologías de la Información y Comunicación (TIC), las cuales son definidas como un grupo de herramientas que apuntan a facilitar el acceso a la información y que a partir de la adquisición de nuevos saberes generan diversos conocimientos. Son, a saber, la internet, softwares, telefonía celular, televisión, radio, entre otras (Corporación Colombia Digital , 2009).

\section{Tecnologías de la comunicación e información TIC}

Las TIC según Belloch (2012), son un conjunto de tecnologías que ayudan a comprender, comunicar y guardar información a través de distintos recursos electrónicos entre los que encontramos videos, ordenadores, teléfonos, y uno de los más importantes, la internet.

En este orden de ideas, el medio o recurso más utilizado a nivel mundial es el ordenador o computador que, acompañado por la internet, permite hacer uso de aplicaciones informáticas, aplicaciones multimedia, programas ofimáticos y redes de comunicación.

El desarrollo tecnológico y de la comunicación ha terciado las distintas plataformas de la vida cotidiana, principalmente la educación y el ámbito laboral. En este orden, el uso de las TIC crea expectativas frente a la mejora de las prácticas pedagógicas por parte de los maestros dado que la implementación de la tecnología en la educación va a promover metodologías de enseñanza renovadas y fundamentadas en el objetivo de construir procesos que apunten a generar nuevos conocimientos y saberes de manera autónoma por el estudiante, fortaleciendo habilidades analíticas y críticas con la guía del docente (Barriga, Flores y Rigo, 2016).

Avendaño, Cortes \& Guerrero, (2015) proponen que utilizar las Tecnologías de la Información y la Comunicación (TIC) le añade un valor agregado al desarrollo de habilidades y competencias en el campo educativo y social, siendo estos aspectos relevantes en tanto se refiere a factores como el desempeño académico en estudiantes de básica primaria y secundaria.

Por otra parte, se plantea que las TIC se consideran una herramienta revolucionaria en la medida en que el contexto educativo se involucra casi siempre en todos los procesos de enseñanza que se llevan a cabo; además, se utilizan de forma transversal, teniendo en cuenta que contribuyen a un desarrollo multimodal por sus diversas propiedades, llegando a ser un instrumento de utilidad para el maestro y su contribución como guía (Rodriguez, Martinez y Lozada, 2009). 


\section{Procesos de comunicación}

\section{escrita y oral}

Dentro de los procesos de comunicación tanto escrita como oral, es importante mencionar que la lectura como una actividad primordial en el proceso de aprendizaje va a permitir al estudiante reconocer, recopilar y estudiar la información que viene del otro y la que se encuentra registrada en los medios actuales de comunicación, además, va a poder interpretar y dar sentido al contenido llegando a transformar sus conocimientos previos y aprehender los nuevos (Ferreiro y Teberosky, 2005).

En este sentido, la lectura como factor relevante en la comunicación escrita y oral, da paso a que el ser humano en general ordene lo aprendido por medio de la deducción y análisis de la información, teniendo en cuenta que el desarrollo de los procesos cognitivos es determinante. Se agrega que, a partir de lo anterior, existe una construcción de saberes referente a como se actúa frente al mundo y como se asumen los conceptos propios y los conceptos de los demás (Teberosky, 2005).

Por lo tanto, la implementación de las TIC en la formas de comunicación escrita $\mathrm{y}$ oral es de gran beneficio debido a que los niños, niñas y jóvenes tienen acceso con más facilidad al manejo de las herramientas que contienen y reproducen la información (internet, computadores, teléfonos celulares), dando paso a una mayor difusión y procesamiento de los contenidos que se encuentran en la web y de las formas de adquirirlos, concluyendo que cada vez las personas puedan transformar su actitud frente a los procesos de aprendizaje y lograr interiorizar conocimientos que van a mejorar de manera directa capacidades y habilidades que le ayudan a desenvolverse en el medio académico (Mujica, 2000).

\section{Metodología}

La presente investigación consta de un diseño metodológico de tipo cualitativo con un alcance descriptivo, y un diseño de campo donde los investigadores recolectaron la información directamente del entorno natural en el que se encuentran los participantes, sin tener control alguno de las categorías de estudio (Arias, 2012). Teniendo en cuenta que se pretende observar y describir cómo la presentación de la serie el Profesor "Súper O" contribuye a mejorar las competencias comunicativas en cuanto a cómo se utiliza el idioma español.

\section{Escenario y actores}

La población participante del estudio corresponde a cincuenta (50) estudiantes de la I.E.D Félix Ospino Mugno, con edades entre 7 y 12 años, quienes cursan grados entre segundo $\left(2^{\circ}\right)$ y cuarto $\left(4^{\circ}\right)$ de primaria.

\section{Técnicas e instrumentos}

En concordancia con el diseño metodológico escogido, se seleccionó como técnica de recolección de datos la observación y como instrumento el diario de campo, en el cual se registraron todos los aspectos que surgían del proceso de investigación, incluyendo el desarrollo de las actividades (Hernandez, 2014) para el registro de la información y los episodios de la serie del profesor Súper O.

\section{Procedimiento}

Las actividades que se desarrollaron en la investigación estuvieron direccionadas a presentar de manera inicial los episodios (3) elegidos de la serie animada del Profesor Súper $O$, específicamente del eje temático de la lucha idiomática. Esta se compone de tres franjas que corresponden a tres ejes temáticos que son: "la lucha por la justicia idiomática" (súper O Idiomático), "la lucha por combatir los yerros históricos" (súper O 
Histórico) y "la lucha y defensa del medio ambiente" (súper O Recargado).

En la transmisión de cada uno de los episodios se observó y describió las actitudes de los estudiantes participantes y se consignaron sus comentarios y reflexiones en el diario de campo, teniendo en cuenta que el objetivo del estudio es mejorar la expresión oral y escrita de los estudiantes en el área de Lengua Castellana. Esta actividad se implementó durante cuatro semanas con la emisión de un episodio por semana y finalmente se realizó una sesión de cierre en la que se llevó a cabo una mesa redonda donde los estudiantes expusieron sus reflexiones acerca de los aprendizajes adquiridos.

\section{Resultados}

Los hallazgos encontrados a partir del desarrollo de las actividades diseñadas para el estudio fueron los expresados en la tabla 1.

TABLA 1

Resultados de la observación en la emisión de los episodios del profesor súper $O$

\begin{tabular}{ll}
\hline $\begin{array}{c}\text { Contenido educativo } \\
\text { del "Profesor Súper Ó" }\end{array}$ & Observaciones \\
\hline
\end{tabular}

Durante la presentación del primer episodio una parte de los estudiantes participantes, la mitad respectivamente, se mostraron ansiosos e interesados por el contenido de la serie, debido a que algunos aseguraban no haberla visto nunca, mientras la otra parte de ellos manifestaban conocer de qué se trata, pero que no la veían frecuentemente. En esta primera proyección del capítulo 01 del Profesor Súper O idiomático se

Episodio 1. "El profesor Súper Ó idiomático 01"

Episodio 2

"el Profesor Súper Ó idiomático 02" trató la temática de las redundancias en las expresiones cotidianas. En el transcurso de la emisión, los estudiantes se observaron atentos y entre ellos realizaban comentarios como "anda mira, eso no se dice así", "mira eso nosotros lo decimos siempre y está mal dicho, hasta lo escribimos mal". Al final de la actividad los estudiantes se vieron motivados para asistir a la próxima sesión y continuar viendo la serie, ya que expresaron que "así no se nos hace aburrido aprender temas de español”, "ojalá y todos los profesores de español dieran las clases con televisión, siento que así aprendo más", "hoy aprendí cómo se dicen y cómo se escriben palabras que siempre utilizo”.

En la emisión del episodio número 2 se trabajó cómo se dicen y se escriben de manera correcta expresiones relacionadas con el verbo hacer. En éste, además, se toca la temática a través del contenido de la nutrición infantil. La totalidad de los estudiantes se mostró interesada en los tópicos del episodio debido a que todos atendieron en el tiempo de la actividad y no hubo distracciones mayores, escuchando comentarios entre ellos como " $\mathrm{ll}$ verbo hacer tiene varios modos y a veces lo escribimos mal", "por favor compañera haz silencio que los que estamos viendo es importante para aprender a escribir y hablar". Al final de la actividad se manifestaron participaciones activas de los estudiantes afirmando tener claridad sobre cómo se dicen y escriben palabras que cotidianamente usan y de aprender expresiones que son de uso frecuente para implementarlas de manera correcta. 


\begin{tabular}{|c|c|}
\hline $\begin{array}{l}\text { Episodio } 3 \\
\text { "el Profesor Súper Ó } \\
\text { idiomático 03”" }\end{array}$ & $\begin{array}{l}\text { En la proyección del tercer episodio los estudiantes se notaron mucho } \\
\text { más motivados en el sentido que llegaron con una alta expectativa sobre } \\
\text { qué les enseñaría el tercer capítulo de la serie. Esto se evidenciaba en } \\
\text { preguntas como "profe, qué aprenderemos de nuevo hoy, me gustaría } \\
\text { saber algo nuevo hoy", "Imagínese profe que he escuchado a mis papás } \\
\text { decir frases que yo sé que están mal y los he podido corregir", "Seño, } \\
\text { quiero saber qué veremos hoy, me emociona saber qué corregiré de mi } \\
\text { hablar". En este episodio la serie explica cómo se nombran y escriben los } \\
\text { números ordinales, plasmando qué expresiones son correctas y cuáles no, } \\
\text { provocando sorpresa en los estudiantes al saber que hay palabras que no } \\
\text { existen y por ende no están bien dichas. }\end{array}$ \\
\hline $\begin{array}{l}\text { Episodio } 4 \\
\text { "el Profesor Súper Ó } \\
\text { idiomático 04" }\end{array}$ & $\begin{array}{l}\text { En la emisión del episodio 04, último para el desarrollo del proyecto, } \\
\text { los estudiantes se mostraron animados en la medida que estaban } \\
\text { curiosos ante qué temática se trabajaría. Este capítulo abordó los tipos } \\
\text { de palabras (palabras parónimas), por lo que los estudiantes quedaron } \\
\text { impactados en la medida en que no sabían acerca de este tipo de } \\
\text { clasificaciones y al conocerlas descubrieron que hay palabras mal dichas, } \\
\text { mal escritas y que no existen. } \\
\text { Manifestaron que es de gran ayuda la muestra de este tipo de programas } \\
\text { porque les ayuda a aprender temas que consideran complicados en } \\
\text { relación con uso de métodos tradicionales de enseñanza. }\end{array}$ \\
\hline $\begin{array}{l}\text { Actividad } \\
\text { de reflexión } \\
\text { "mesa redonda" }\end{array}$ & $\begin{array}{l}\text { En esta actividad se organizó a los estudiantes en un salón de clases } \\
\text { en posición de mesa redonda para socializar sus experiencias a partir } \\
\text { de la visualización de la serie del Profesor Súper Ó. Los estudiantes } \\
\text { expresaron ideas tales como: "me pareció que la actividad de pasar la } \\
\text { serie para aprender temas de español de una forma divertida, es muy } \\
\text { buena, porque generalmente los profesores dictan las clases y hablan y } \\
\text { ya, y sentí que así se aprende más y uno presta más atención", "profe, } \\
\text { yo creo que la manera de aprender español no debe ser solo en el salón y } \\
\text { con el libro y el cuaderno a mí me gustó mucho porque siempre teníamos } \\
\text { la oportunidad de aprender de una forma entretenida”, "Seño, a mí me } \\
\text { gustó porque me reía y aprendía a la misma vez, y de temas que son } \\
\text { importantes para mejorar la lectura y escritura". En esta sesión los } \\
\text { estudiantes estuvieron animados, participativos y receptivos atendiendo } \\
\text { las reflexiones de todos los compañeros. }\end{array}$ \\
\hline
\end{tabular}

Fuente: elaboración propia.

\section{Discusión}

A partir de la elaboración del proyecto de investigación los docentes identificaron que los estudiantes lograron aprender temáticas propias de la Lengua Castellana, de una manera didáctica, desde la implementación de la serie del Profesor Súper Ó como una estrategia televisiva que promueve el correcto uso del lenguaje español, propiciando una transformación en la percepción de los estudiantes acerca de cómo se utilizan determinadas expresiones, cómo se escriben y cómo se leen; además, permitiendo que éstos se empoderen de los procesos de aprendizaje autónomo y denoten los nuevos métodos de enseñanza que utilizan los maestros, tratando de responder a las nuevas exigencias de la educación. Tal como lo plantean Rodriguez, Martinez, \& Lozada (2009), las TIC en el contexto educativo van a permitir que los procesos de enseñanzaaprendizaje se realicen de manera transversal e interdisciplinar, logrando que el maestro identifique las herramientas necesarias para apoyar a los estudiantes y brindar el apoyo como guía del desarrollo académico. 
Los estudiantes se mostraron motivados en la dinámica de aprender e interiorizar conocimientos nuevos, lo cual será el punto inicial para trazar estrategias que promuevan el estudio de la Lengua Castellana con el fin de potenciar capacidades y habilidades que en un futuro contribuyan al desenvolvimiento tanto en la vida escolar como en la universitaria. Considerando la postura de Mujica (2000), el uso de estrategias pedagógicas innovadoras como las TIC, contribuye a que los estudiantes tengan un mayor procesamiento de información y por ende de adquisición de conocimientos que van a cambiar su actitud frente a las formas de aprendizaje y cómo éstas le fortalecen su desarrollo en el ámbito educativo.

Esta es una de las razones por la la cual los autores Mendoza, López y Salas (2016), señalan que las organizaciones "son entes que generan sus propios objetivos y diseñan la forma como alcanzarlos teniendo en cuenta, necesariamente, las influencias del entorno social" (p. 62). Las escuelas no escapan de ello y es de allí que, en las organizaciones cualquiera que sea su ámbito, se hizo preciso introducir esta estrategia. A lo que se le podría sumar lo dicho por Arellano (2004) citado por Mendoza y López (2015), haciendo referencia a la forma del pensamiento donde expresa que este "se dirige hacia la creatividad, imaginación, y búsqueda de opciones innovadoras y realistas, como potenciación de lo posible enmarcado dentro de un proyecto de construcción intencional de la realidad" (p. 154).

Finalmente, los maestros proponen que el uso correcto del lenguaje Español da cabida para que se estudien de forma transversal temáticas de carácter interdisciplinar, apoyándose en la implementación de métodos de enseñanza innovadores que incluyan el uso de las tecnologías de la información y comunicación (TIC) para así enriquecer el proceso y mantener el entusiasmo en la población estudiantil, obteniendo también otros espacios para impulsar la investigación como una estrategia pedagógica permanente en el plan curricular.

\section{Referencias}

Alba, D. y Gómez, D. (2015). La construcción multimodal del signo ideológico "idioma" en la serie animada de televisión colombiana, El profesor Súper Ó. ANTARES: Letras e Humanidades, 7(14), 271-296.

Arceo, F. , Rojas, G. y González, E. (2010). Estrategias docentes para un aprendizaje significativo: una interpretación constructivista (Vol. 2). México, D.F.: McGraw Hill.

Avendaño, I., Cortés, O. y Guerrero, H. (2015). Competencias sociales y tecnologías de la información y la comunicación como factores asociados al desempeño en estudiantes de básica primaria con experiencia de desplazamiento forzado. Diversitas, 11(1), 13 36 .

Belloch. (2012). Las Tecnologías de la Información y Comunicación en el aprendizaje. Recuperado de https://www. uv.es/bellochc/pedagogia/EVA1.pdf

Botello, S. (2013). La escritura como proceso y objeto de enseñanza. [Tesis de maestría]. Universidad de Tolima, Ibagué.

Carbó, C., Llisterri, J., Machuca, M., Mota, C., Riera, M. y Ríos, A. (2003). Estándar oral y enseñanza de la pronunciación del español como primera lengua y como lengua extranjera. ELUA. Estudios de Lingüística, 17(1). 161-179.

Camps, A. (2004). Objeto, modalidades y ámbitos de la investigación en didáctica de la lengua. Revista LENGUAJE, 32. 7-27. 
Cedeño, S. (2015). La lúdica una estrategia pedagógica en la construcción de valores para favorecer procesos de enseñanza-aprendizaje en los niños de 4-5 años en la institución educativa San Simón de Ibagué. [Tesis grado]. Universidad de Tolima, Ibagué.

Ciprian, J. (2012). La investigación como estrategia pedagógica de construcción de ciudadanía participativa para niños, niñas y jóvenes del programa ondas. [Tesis Magistral]. Universidad Pedagógica Nacional. Bogotá, D.C.

Cortés, M. (2005). ¿Hay que enseñar gramática a los estudiantes de una lengua extranjera? Cauce, (28): 89-108.

Deleito, P., Llera, J. y Cobeñas, E. (2009). Perfil en estrategias de aprendizaje de estudiantes de alto rendimiento en lengua castellana y literatura. Faísca: revista de altas capacidades, 14(16), 49-75.

Fernández-Arata, J. M. (2008). Desempeño docente y su relación con orientación a la meta, estrategias de aprendizaje y autoeficacia: un estudio con maestros de primaria de Lima, Perú. Universitas Psychologica, 7(2). 385-401.

Feria, A. y Martínez, I. (2011). Estrategia de aprendizaje de los contenidos de la disciplina fundamentos pedagógicos de la educación para el perfeccionamiento de la formación inicial de los estudiantes que cursan el primer año en la licenciatura en educación carrera pedagogía psicología. Cuadernos de Educación y Desarrollo, 3(28). Recuperado de http://www.eumed.net/rev/ ced/28/ofmm.htm

Franco, M. (2012). Estrategias de enseñanza para la promoción de la comprensión lectora desde el aprendizaje significativo. Cultura, Educación, Sociedad, 3(1), 175-186.
Hanssen, F. y Alfonso, L. (1913). Gramática histórica de la lengua castellana. Buenos Aires: El Ateneo.

Herrera-Mendoza, K., Acuña, M., y Gil, L. (2017). Motivación de jóvenes hacia el uso de teléfonos celulares. Encuentros, 15(1). 91-105.

Herrera, B. (2013). La constitucionalización de los derechos del consumidor en Colombia: un análisis desde los derechos sociales fundamentales. Civilizar. Ciencias Sociales y Humanas, 13(25), 33-47.

Herrera-Tapias, B. (2016). Cultura Ciudadana y las Tecnologías de la Información y la Comunicación. Barranquilla: Editorial YOYOBIZ.

Marín, F., Niebles, M., Sarmiento, M. y Valvuena, S. (2017). Mediación de las tecnologías de la información en la comprensión lectora para la resolución de problemas aritméticos de enunciado verbal. Revista Espacios, 38( $\left.\mathrm{N}^{\circ} 20\right)$. Recuperado de http://www.revistaespacios.com/a17v38n20/a17v38n20p20.pdf

Marinkovich, J. (2007). Las estrategias cognitivo-retóricas y la dimensión dialéctica de la argumentación oral en una clase de lengua castellana y comunicación. Signos, 40(63), 127-146.

Mendoza, D. y López, D. D. (2015). Pensamiento estratégico: centro neurálgico de la planificación estratégica que transforma la visión en acción. Económicas CUC, 36(1), 153-179.

Mendoza, D., López, D. y Salas, E. (2016). Planificación estratégica de recursos humanos: efectiva forma de identificar necesidades de personal. Económicas CUC, 37(1), 61-79.

Monereo, C., Castelló, M., Clariana, M., Palma, M. y Pérez, M. L. (2000). Estrategias de enseñanza y aprendizaje. Barcelona. Editorial Graó. 
Navarro, J. (2003). Inmigración en España y conocimiento de la lengua castellana. El caso de los escolares inmigrados en Aragón. [Tesis doctoral]. Universitat de Lleida, Lleida.

Núñez, J., González, J., García, M., González, S., Roces, C., Álvarez, L. y González, M. (1998). Estrategias de aprendizaje, autoconcepto y rendimiento académico. Psicothema, 10(1). 97-109.

Prado, J. (2001). La competencia comunicativa en el entorno tecnológico: desafío para la enseñanza. Comunicar, (17). 2130.

Pinzón, L. (2011). ¿Qué dejó a la historiografía regional el Bicentenario de la Independencia de Colombia? La resignificación del Socorro y los socorranos. Anuario de Historia Regional y de las Fronteras, 16(1), 331-352.

Pozo, J. y Gómez, M. (2006) Aprender y enseñar ciencia. 5ta. Edición. Madrid: Ediciones Morata.
Reyzábal, M. (2012). Las Competencias Comunicativas y Lingüísticas, clave para la calidad educativa. Reice. Revista Iberoamericana sobre Calidad, Eficacia y Cambio en Educación, 10(4), 63-77.

Sáez, F. (2002). Objetivos en la enseñanza de lenguas extranjeras: De la competencia lingüistica a la competencia intercultural. Inmigración, Convivencia e Interculturalidad. Ceuta: Instituto de Estudios Ceutíes.

Torres, Y. (2017). Análisis pedagógico de las teleclases de 1970 y la serie el Profesor Super O, desde las políticas de televisión educativa. [Tesis doctoral]. Universidad Pedagógica Nacional, Bogotá, D.C.

Zavala, I. M. (1993). Breve historia feminista de la literatura española (en lengua castellana) (Vol. 6). Barcelona: Anthropos Editorial. 